Sādhanā Vol. 40, Part 3, May 2015, pp. 1023-1031. (C) Indian Academy of Sciences

\title{
Dynamics of a spreading thin film with gravitational counterflow using slip model
}

\author{
NAVEEN TIWARI \\ Department of Chemical Engineering, Indian Institute of Technology Kanpur, \\ Kanpur, UP 208016, India \\ e-mail: naveent@iitk.ac.in
}

MS received 10 July 2014; revised 22 January 2015; accepted 6 February 2015

\begin{abstract}
Thin liquid films can be made to spread along a solid surface by application of temperature gradients at the liquid-gas interface. The surface tension of usual liquids decreases linearly with temperature thus producing the driving thermocapillary stresses due to the applied temperature gradient. These spreading films are susceptible to a fingering instability at the advancing solid-liquid-vapor contact line, which is linked to the development of a capillary ridge near the advancing front. A thin film climbing up on a vertical substrate against gravity shows interesting dynamics due to strong opposing gravitational counterflow. At the contact-line of the spreading film, slip-model is used to alleviate the stress-singularity due to more usual no-slip boundary condition. It is shown that depending upon the magnitude of a gravitational drainage parameter the steady-profiles of the spreading films show qualitatively different dynamics. The dynamics is in agreement with the experimentally observed profiles in the literature as well as computed profiles using precursor-film model at the contact-line in some earlier theoretical studies. Briefly, their stability behavior is also discussed.
\end{abstract}

Keywords. Slip-model; thermocapillary flow; drainage.

\section{Introduction}

Thin liquid films spreading on a solid substrate has been a topic of research for past several decades. One fundamental question that researchers have tried to address is the stress-singularity at the solid-liquid contact-line that arises due to more commonly used no-slip boundary condition. Three most common methods to relieve stress-singularity while modeling spreading phenomena are slip-model, precursor film model, and introduction of explicit intermolecular forces at the liquid-solid contact line. Detailed explanation of these methods can be found in the classical work of Huh \& Scriven (1971). A recent review article by Kirkinis \& Davis (2013) discussed the numerical methods to implement these models for moving contact lines. It is important to note that the precursor film model is applicable for perfectly wetting liquids where as slip-model can accommodate partially wetting liquids. 
In the present work, dynamics of a thin film spreading due to a thermocapillary stress is modeled within lubrication approximation. In microscale flows, the large surface to volume ratios allow interfacial stresses to exert a driving influence. This ability to drive thin film using thermocapillary stress is used to spread film for micro-fabrication related applications. The dynamics of such flows has been studied extensively for example see Kataoka \& Troian (1997), Davis \& Troian (2003), Davis et al (2003), Bertozzi et al (1998) and Grigoriev (2003). The thermal gradient applied to a thin liquid film on a solid substrate can induce a thermocapillary shear stress due to the variation, mostly assumed linear, of surface tension with temperature. It has also been noted that thin films of Newtonian liquid when driven to spread on flat, homogeneous surfaces by these stresses are susceptible to a fingering instability at the solid-liquid-vapor contact line. Linear stability analysis for example by Davis \& Troian (2004) has shown that the instability is linked to the development of a capillary ridge near the advancing front.

For the spreading of a thin film several theoretical studies have shown quantitative agreement between the base state profiles and dispersion curves obtained using precursor-film and slip model for thin films driven solely by an applied shear stress (Davis 2003) or body force (Spaid \& Homsy 1996). Computed predictions for the wavelength and growth rate of the instability are generally in good agreement with experimental data. There has been considerable work on the dynamics of thermocapillary spreading films with competing gravitational counterflows (Bertozzi et al 1998, 1999; Davis et al 2006), and the precursor film model has been used in most of these studies (Kataoka \& Troian 1998; Bertozzi et al 1998, 1999). With two competing forces namely the thermocapillary stress and gravity, complex wave form solutions are obtained that evolve in time and space. In addition to the usual compressive shocks that appear in the problem involving only one driving force, these films also exhibit "undercompressive" traveling wave solutions that have been observed in experiments. In addition solutions with simultaneous compressive and undercompressive waves are also found that propagate at different velocities as shown by Bertozzi et al $(1998,1999,2001)$. Furthermore, it has been shown by Kataoka \& Troian (1998) using a precursor film model that a sufficiently large gravitational counterflow suppresses the capillary ridge and produces a linearly stable film.

In this paper, the dynamics of thermocapillary spreading films is analyzed using the Greenspan slip model (Greenspan 1978) for the entire range of gravitational drainage, beginning at zero and increasing until it balances the thermocapillary stress and a stationary film profile is attained. In addition, effect of dynamic contact angle is considered on the solution forms. The dependence of the contact angle on slip-velocity of the contact-line is similar to that used by Lopez et al (1996). Importance of more degrees of freedom in slip-model is demonstrated as compared to the precursor film model in the context of the film dynamics involving to competing forces.

\section{Problem formulation}

A thin film of a Newtonian liquid of density $\rho$, viscosity $\mu$, and surface tension $\sigma$ that is driven to spread by thermocapillary stresses along a planar vertical surface. The schematic is shown in figure 1(a). The fingering instability at the spreading front associated with such film spreading problems is shown in figure 1(b). A decreasing linear temperature profile is imposed on the solid substrate such that $d T / d x<0$ where $T$ is the temperature at the liquid-gas interface. Furthermore, the Biot number is assumed very small such that the temperature at the liquid-air interface is essentially the same as that imposed on the solid substrate which is a valid assumption for such thin films. The surface tension, $\sigma$, for most liquids has linear dependence on $T, \sigma(T)=$ $\sigma_{0}-\gamma\left(T-T_{0}\right)$, where $\gamma=d \sigma / d T>0$ is a constant (e.g. see Davies \& Rideal 1963). The 


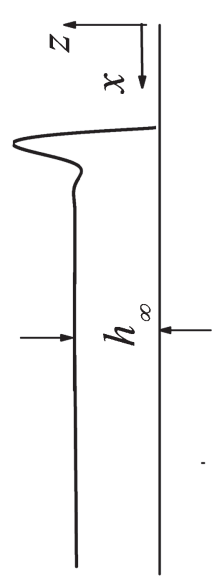

(a)

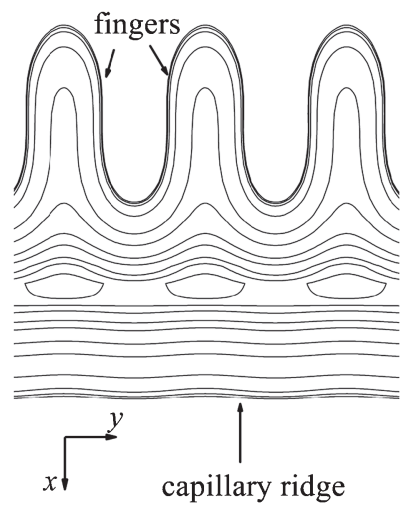

(b)

Figure 1. (a) Schematic of a thin liquid film climbing up a vertical substrate in the $-x$-direction due to thermocapillary stress; (b) Schematic of fingering instability developing at the three-phase contact line.

applied temperature gradient imposes a thermocapillary stress $\tau=-d \sigma / d x$ at the liquid-air interface, which forces the film to spread up the inclined plane in the $-x$-direction. It is assumed that the liquid is supplied from the reservoir at constant flux. The $z$-direction points outwardly normal from the substrate, and $y$ is the transverse coordinate in the plane of the substrate. The local film thickness is $h$, and $h_{\infty}$ is the constant film-thickness away from the spreading edge of the film where capillary effects are negligible. Note that the physical properties are evaluated at the reference temperature $T_{0}$ in the dimensionless groups introduced below.

Within the lubrication approximation and assuming that nothing happens in $y$-direction the Navier-Stokes equation and continuity equation can be simplified to give the film evolution equation as,

$$
h_{t}-\left[\frac{\tau h^{2}}{2 \mu}\right]_{x}+\left[\frac{\rho g}{3 \mu}\left(h^{3}+\alpha h\right)\right]_{x}+\left[\frac{1}{3 \mu}\left(h^{3}+\alpha h\right)\left(\sigma h_{x x}\right)_{x}\right]_{x}=0 .
$$

At the solid boundary, the well-known Greenspan slip-model (Greenspan 1978) is applied to alleviate the stress-singularity at the contact line,

$$
\left.u\right|_{z=0}=u_{s}=\left.\frac{\alpha}{3 h} \frac{\partial u}{\partial z}\right|_{z=0},
$$

where $\alpha$ is the dimensional slip-coefficient. Taking $\alpha / h_{\infty}^{2} \ll 1$ restricts slip to a small region near the moving contact line.

Equation (1) is made non-dimensional near the contact line by using the dynamic capillary length, $l_{c}=h_{\infty} /(3 \mathrm{Ca})^{1 / 3}$ as the characteristic length in the $x$-direction, and $h_{\infty}$ as the characteristic length scale in the z-direction. The capillary number is $\mathrm{Ca}=\mu U_{c} / \sigma_{0}$, where $U_{c}=h_{\infty} \tau / 2 \mu$ is the characteristic velocity of the thermocapillary flow.

After rescaling the variables, Eq. (1) becomes

$$
h_{t}-\left(h^{2}\right)_{x}+B\left(h^{3}+\alpha h\right)_{x}+\left[\left(h^{3}+\alpha h\right)\left(h_{x x x}\right)\right]_{x}=0,
$$


where $B=2 \rho g h_{\infty} / 3 \tau$ is the non-dimensional parameter that represents the importance of drainage due to gravity relative to the thermocapillary stress.

The solid-liquid-vapor contact line is located at $x=a(y, t)$, which gives the condition

$$
h(x=a(y, t))=0 .
$$

At the contact line, a dimensionless relationship between the dynamic contact angle, $\theta=$ $\partial h / \partial x(x=a(y, t))$, and the contact line velocity, $a_{t}$, is assumed which is similar to that used in the theoretical study of non-isothermal spreading of liquid films on an inclined plane by Lopez et al (1996),

$$
h_{x}(x=a(y, t))=\theta_{s}+D\left(-a_{t}\right)^{1 / 3},
$$

where $D=\left(U_{c} / k\right)^{1 / 3}\left(l_{c} / h_{\infty}\right)$ is a dimensionless parameter quantifying the dependence of the contact angle on the velocity of the contact line, $\theta_{s}$ is the static contact angle and $k$ is a fitted positive constant.

As $D \rightarrow 0$, the motion of the contact line does not affect the contact angle, which corresponds to the limit of a fixed contact angle that was used in most previous studies for example by Davis \& Troian (2004) and Grigoriev (2003).

The static reference frame is transformed into one that moves with the contact line velocity by introducing

$$
\zeta(x, t)=x-a(t),
$$

such that $\zeta=0$ corresponds to the location of the contact line. In the moving reference frame, Eq. (1) becomes

$$
h_{t}-a_{t} h_{\zeta}-\left(h^{2}\right)_{\zeta}+B\left(h^{3}+\alpha h\right)_{\zeta}+\left[\left(h^{3}+\alpha h\right) h_{\zeta \zeta \zeta}\right]_{\zeta}=0 .
$$

For a steady traveling wave, solution $h_{t}=0$ and therefore Eq. (7) can be simplified to

$$
h_{\zeta \zeta \zeta}=-B+\frac{h+a_{t}}{h^{2}+\alpha},
$$

For a steady traveling-wave, the constant wave speed can be found by realizing that at $\zeta \rightarrow \infty$ derivatives become zero and film-thickness is unity. Thus the constant wave speed is given by

$$
a_{t}=-1+B(1+\alpha)
$$

The boundary conditions at the contact line given by Eqs. (4)-(5) are rewritten for the base profiles as (Lopez et al 1996)

$$
h(\zeta=0)=0, h_{\zeta}(\zeta=0)=\theta_{s}+D\left(-a_{t}\right)^{1 / 3} .
$$

The boundary conditions far from the contact line correspond to a flat film,

$$
h(\zeta \rightarrow \infty)=1, h_{\zeta}(\zeta \rightarrow \infty)=0
$$

As will be discussed in the next section under some conditions, it is not possible to find steady traveling-wave solution. For such case, the transient term in Eq. (7) cannot be dropped and therefore an unsteady equation will be solved along with the above-mentioned boundary conditions. 


\section{Results and discussion}

The traveling-wave solution using Eq. (8) was found by a shooting method with fourth-order Runge-Kutta scheme. In figure 2 the parameter space division is shown based on the qualitatively different profiles for film-thickness, $h(\zeta)$, depending on the strength of gravitational drainage. In region-I steady traveling-wave solutions could be found for all values of static contact angle $\theta_{s}$. As drainage parameter $B$ is increased region-II is attained in which traveling-wave solution could not be found and unsteady film-profiles were obtained. In region-III (large $B$ ) steady traveling-wave solutions were found but for some specific values of the static contact angle. These qualitatively different film-thickness profiles corresponding to the three regimes are shown in figure 3.

The film-thickness base profiles are shown in figure 3 for slip-coefficient $\alpha=0.1$, staticcontact angle $\theta_{s}=0.1$ and $D=0.1$. For this combination of parameters of the slip-model, $B_{L}=0.22$ and $B_{U}=0.685$ which can found from figure 2. $B_{L}$ and $B_{U}$ define the lower and upper limits respectively in the parameter space within which traveling-wave solutions cannot be found. In figure 3, the film profiles for $B=0.1$ and $B=0.2\left(B<B_{L}\right)$ are steady traveling-wave profiles. It is evident that as $B$ is increased from 0.1 to 0.2 the height of the capillary ridge near the moving contact line increases. This increase in height is due to the opposing gravitational drainage indicated by the increase in value of $B$. In the work of Lopez et al (1996) gravity driven films were considered and it was found that larger capillary ridge (which was due to larger contact-angle in their case) was more unstable to fingering instability. For a thermocapillary driven film with opposing gravity steady traveling-wave solutions were also found by Bertozzi et al (1998) but using precursor film model at the contact-line. They had also observed a similar increase in height of the capillary ridge with increase in drainage (though their scaling methods were different than used in this study making a direct quantitative comparison not possible). They had found that increase in capillary ridge leads to stabilization of the ridge which was contrary to the behavior of gravity driven films such as that in the work of Lopez et al (1996).

An important parameter of the slip-model used in this study is $D$ that relates the motion of the contact-line to the apparent contact-angle. The relation is shown in Eq. (5). For a non-zero value of $D$, the apparent contact-angle is higher than the static contact-angle. It was shown in the work of Lopez et al (1996) that as the value of $D$ increased (implies increase in apparent

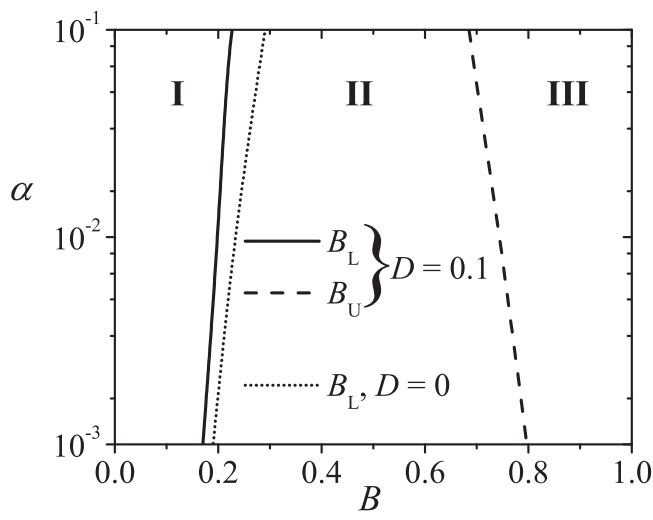

Figure 2. Division of the parameter space in drainage parameter for slip-model. The nature of the base profile varies significantly in these solution regimes. 
contact-angle) keeping all parameters of slip-model same the behavior of the film profile changes and becomes more unstable to transverse (in $y$-direction) perturbations. Similarly, $D$ is found to have significant effect on the behavior of film profiles in this study. One curve for $B_{L}$ for $D=0$ with all other parameters same $\left(\alpha=0.1, \theta_{s}=0.1\right)$ is shown in figure 2 . It can be noted that with decrease in $D$ (decrease in apparent contact-angle) region-I shifts more to higher values of $B$. Another perspective is that as $D$ or the apparent contact angle increases, the size of region-I shrinks (moves to smaller values of $B$ ).

For region-II, i.e. $B_{L}<B<B_{U}$, a non-traveling-wave solution was found which is inherently unsteady. One such profile at a particular time instant is shown in figure 3 for $B=0.5$. Other parameter values are mentioned in the figure. In this regime, two ridges develop, one near the contact-line and the second one rolls down due to gravity. Similar two waves were found experimentally and theoretically using precursor film model in the work of Bertozzi et al (2001). They called the ridge near contact-line as under-compressive shock and the trailing wave which rolls down as compressive shock. The stability analysis of such a structure showed that the compressive shock was linearly unstable but much less stable (smaller eigenvalues) than the traveling-wave solutions found in region-I. The instability for these unsteady structures was located at the trailing compressive shock. To perform the linear stability analysis, the traditional method of forming a stability matrix operator that depends on the 2D base-profile is performed but with the base-profile "frozen" at a time instant for example the one shown in figure 3. The other method is to solve the unsteady base-profile equation along with the linear stability equation in time and track the growth-rate of a random initial perturbation.

For region-III, i.e. $B>B_{U}$, a traveling-wave solution was found again but only for a fixed value of $\theta_{s}$ for given values of $\alpha$ and $D$. For other values of $\theta_{s}$, unsteady non-traveling solutions were found. Curves that represent the value of $\theta_{s}$ at each $B$ (larger than $B_{U}$ ) for fixed $\alpha$ and $D=0.1$ for which steady film profiles were found as shown in figure 4 . One such film-profile for $B=0.75$ is shown in figure 3 . The moving front in this region is monotonically decreasing from a constant film-thickness to zero at the contact-point. Thus only the under-compressive shock exists for this region. Using a precursor film model and experiments, Sur et al (2003) showed that this under-compressive shock is linearly stable to transverse perturbation. In the work of Kataoka \& Troian (1998), it was shown that for a precursor film model and $B>B_{U}$ there is only one value of precursor film thickness that leads to the steady traveling-wave solution. This

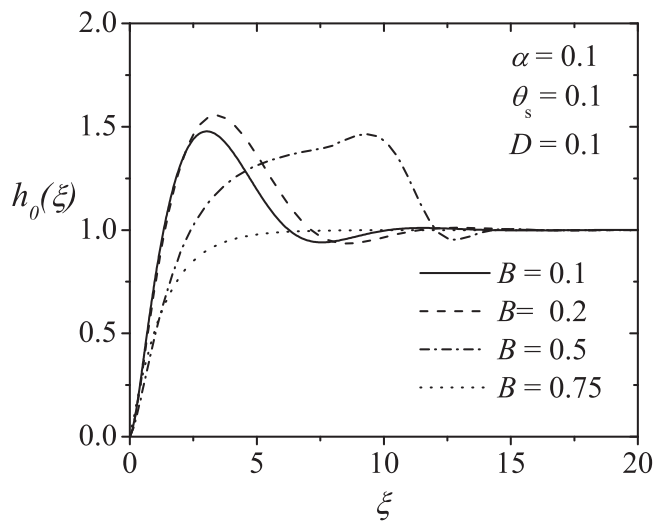

Figure 3. Computed base-profiles for different drainage values. The different form of solutions correspond to the regime found in figure 2. 


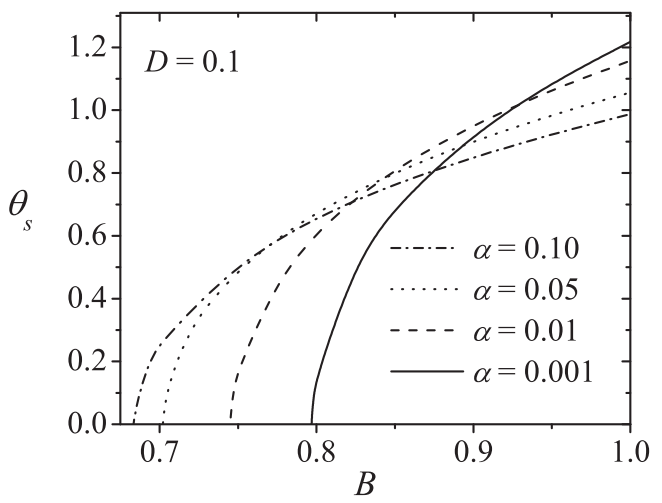

Figure 4. Specific values of $\theta_{S}$ for which traveling-wave solution exists in regime-III (figure 2) when $D=-0.1$.

is in contrast to the slip model results presented here in which steady solutions could be found for any value of slip-coefficient $\alpha$ but $\theta_{s}$ cannot be fixed independently. This fact highlights the wider applicability of the slip-model due to more degrees of freedom. Overall it is interesting to note that as the value of $B$ is increased from region-I through region-III the film profile attains qualitatively different shapes. Furthermore, it is evident that the gravitational drainage has a stabilizing effect on the film.

The drainage parameter $B$ is the important parameter of this study. Therefore, a brief discussion on the typical magnitude and its dependence on other factors is now presented. Munch (2002) theoretically derived the asymptotic thickness (away from the advancing front) of the film spreading due to thermocapillary stress above the meniscus. As per the prediction, the film-thickness $h_{\infty}$ was proportional to the square of the applied stress $\tau^{2}$. Furthermore, the filmthickness was found to be inversely proportional to $C_{0}^{3}$ where $C_{0}$ is the curvature of the meniscus. The result was an extension of some of the earlier previous theoretical studies (Carles \& Cazabat 1993; Fanton et al 1996) and showed very good agreement with experimental data (Fanton et al 1996). In all of these theoretical studies because $h_{\infty} \propto \tau^{2}$, so $B \propto \tau$ and larger thermal gradients correspond to larger $B$ and an increased effect of drainage for the thicker films. Several other experiments of Cazabat et al (1990) and Fanton et al (1996) had found film-thickness corresponding to $0.01<B<0.11$. Therefore, these studies were found to belong to region I in which gravitational drainage has a small effect on the film dynamics. Much larger values of $B$ were obtained in the early experiments of Ludviksson \& Lightfoot (1971). The larger values can be attributed to the experimental method of preparing the films. A film was first dip-coated onto the plate by withdrawing it from a liquid reservoir, and the temperature gradient was then applied, which resulted in a film thickness different than predicted by Munch (2002), and therefore large values of $B$.

\section{Conclusion}

The effect of gravitational drainage on the dynamics of thermocapillary spreading films was explored in this work using a lubrication-based model. The three-phase contact-line was allowed to slip due to which the contact angle $\theta$ and slip coefficient $\alpha$ were introduced into the model as parameters. The dynamic contact angle was considered in which contact angle was allowed 
to depend on the slip-velocity of the contact line. Depending on the magnitude of gravitational drainage compared to the driving thermocapillary force (represented by drainage parameter $B$ ) three qualitatively different spreading regimes were found. The two critical values of this parameter that separate these regions are denoted by $B_{L}$ and $B_{U}$, with $0<B_{L}<B_{U}<1$. For $B<B_{L}$, corresponding to small drainage, a capillary ridge forms at the contact-line. For this regime, $\theta$ and $\alpha$ can be specified independently, and the base states are steady, traveling-wave solutions. For $B>B_{L}$ the film shape is no more a steady traveling-wave solution but evolves continuously in time. For $B \geq B_{U}$, traveling wave solutions can be found, but only for one particular combination of $\alpha$ and $\theta$ for each $B$. The film profile in this region shows monotonically decreasing spreading front. The results generally agree well with the widely used precursor film model in the literature. For precursor film model, there is only one traveling solution for each $B \geq B_{U}$ which is not the case with slip-model due to more adjustable parameters in the slip-model.

\section{References}

Bertozzi A L, Munch A, Fanton X and Cazabat A M 1998 Contact line stability and 'undercompressive shocks' in driven thin film flows. Phys. Rev. Lett. 81: 5169

Bertozzi A L, Munch A and Shearer M 1999 Undercompressive shocks in thin film flows. Physica D 134: 431

Bertozzi A L, Munch A, Shearer M and Zumbrun K 2001 Stability of compressive and undercompressive thin film travelling waves. Eur. J. Appl. Math. 12: 253

Carles P and Cazabat A M 1993 The thickness of surface-tension-gradient-driven spreading films. J. Colloid Interface Sci. 157: 196

Cazabat A M, Heslot F, Troian S M and Carles P 1990 Fingering instability of thin spreading films driven by temperature gradients. Nature 346: 824

Davies J and Rideal E 1963 Interfacial phenomena. New York: Academic Press

Davis J M 2003 Dynamics and linear stability of thermocapillary spreading films on homogeneous and micropatterned surfaces. $\mathrm{PhD}$ thesis, Princeton University

Davis J M and Troian S M 2003 Influence of attractive van der waals interactions on the optimal excitations in thermocapillary driven spreading. Phys. Rev. E 67: 016,308

Davis J M and Troian S M 2004 Influence of boundary slip on the optimal excitations in thermocapillary driven spreading. Phys. Rev. E 70: 046,309

Davis J M, Fischer B J and Troian S M 2003 A general approach to the linear stability of thin spreading films. In: Narayanan R (ed) Interfacial fluid dynamics and transport processes, Lecture Notes in Physics, Springer-Verlag Heidelberg, New York, pp 79-106

Davis J M, Kataoka D E and Troian S M 2006 Transient dynamics and structure of optimal excitations in thermocapillary spreading: Precursor film model. Phys. Fluids 18: 092,101

Fanton X, Cazabat A M and Quéré D 1996 Thickness and shape of films driven by a marangoni flow. Langmuir 12: 5875

Greenspan H 1978 On the motion of a small viscous droplet that wets a surface. J. Fluid Mech. 84: 125

Grigoriev R O 2003 Contact line instability and pattern selection in thermally driven liquid films. Phys. Fluids 15: 1363

Huh C and Scriven L E 1971 Hydrodynamic model of steady movement of a solid / liquid / fluid contact line. J. Colloid Interface Sci. 35: 85

Kataoka D E and Troian S M 1997 A theoretical study of instabilities at the advancing front of thermally driven coating films. J. Colloid Interface Sci. 192: 350

Kataoka D E and Troian S M 1998 Stabilizing the advancing front of thermally driven coating films. J. Colloid Interface Sci. 203: 335 
Kirkinis E and Davis S H 2013 Hydrodynamic theory of slippage on a solid substrate near a moving contact line. Phys. Rev. Lett. 110: 234,503

Lopez P G, Miksis M J and Bankoff S G 1996 Non-isothermal spreading of a thin liquid film on an inclined plane. J. Fluid Mech. 324: 261

Ludviksson V and Lightfoot E N 1971 The dynamics of thin liquid films in the presence of surface-tension gradients. AICHE J. 17: 1166

Munch 2002 The thickness of a marangoni-driven thin liquid film emerging from a meniscus. SIAM J. Appl. Math. 62: 2045

Spaid M A and Homsy G M 1996 Stability of newtonian and viscoelastic dynamic contact lines. Phys. Fluids 8: 460

Sur J, Bertozzi A L and Behringer R P 2003 Reverse undercompressive shock structures in driven thin film flow. Phys. Rev. Lett. 90: 126,105 